\title{
LXI. The magnetic circuit of dynamo machines
}

\section{W.E. Ayrton \& John Perry}

To cite this article: W.E. Ayrton \& John Perry (1888) LXI. The magnetic circuit of dynamo machines, Philosophical Magazine Series 5, 25:157, 496-510, DOI: 10.1080/14786448808628219

To link to this article: http://dx.doi.org/10.1080/14786448808628219

$$
\text { 曲 Published online: } 29 \text { Apr } 2009 .
$$

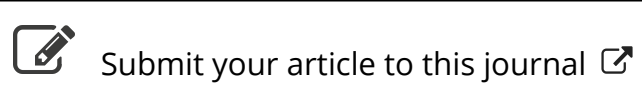

Џلl Article views: 3

Q View related articles 두 
axis. The explanation of this seems almost certainly to be an increase in the resistance of the liquid column, owing to the presence in considerable quantity of the gaseous products in the path of the current. A straight line dotted thus ...... has been ruled through those points which lie on the straight part of the curve, to cut the axis of potential. Representing this straight line by the equation $\mathrm{E}=e+\mathrm{CR}$, and putting in the numerical values of $\mathrm{E}$ and $\mathrm{C}$, a series of equations was obtained containing $e$ and $\mathrm{R}$. The mean value of $e$ at $40^{\circ}$ is $2 \cdot 32$, and at $80^{\circ} 2 \cdot 08$; and these values of the intercept on the axis of potential represent in volts the values of the maximum steady electromotive force of polarization at these temperatures under the conditions of the experiment. The difference $\cdot 24$ volt, produced by a rise of $40^{\circ}$ in temperature, gives $\cdot 26$ per cent. as the rate of variation per degree at temperature $40^{\circ}$. The other constant $R$ of the straight line, representing the tangent of its angle of inclination to the axis of current, being the quotient of potential by current, indicates the resistance of the voltameter under the conditions of the experiment. The mean values of $R$ obtained from the equations were for $40^{\circ}, \cdot 69$, and for $80^{\circ}, 67$; these numbers representing in ohms the working resistance of the voltameter.

Conclusions.-(1) The opposing electromotive force of polarization which arises in cells when at work depends on the value of the current passing through them when that current is below a certain value, increasing, but more and more slowly, with the current.

(2) There is a maximum value of the polarization regarded only as a function of the current-strength, beyond which any increase in the strength of the current has no effect upon it.

(3) The electromotive force of polarization varies with temperature, its value decreasing about 1 per cent. for a rise of temperature of $4^{\circ} \mathrm{C}$.

The method of experiment was suggested by Professor Carey-Foster, in whose laboratory the experiments were made.

LXI. The Magnetic Circuit of Dynamo Machines. By W. E. Ayrton and John Perry.*

[Plate IV.]

T $\mathrm{N}$ this paper we shall ase the following symbols :1 Dimensions are given in centimetres, current in amperes, potential differences and electromotive forces in volts, resistances in ohms

* Communicated by the Physical Society : read March 10, 1888. 
We shall speak indifferently of the drum or Hefner-Alteneck and the cylindric Gramme armature-that is, the Gramme armature which receives its induction through its cylindric surface from two pole-pieces. It is easy to make the slight changes in the formulæ required for flat gramme ringarmatures like those of the Victoria, Gulcher, and Schückert machines, which receive induction through their flat sides, and for all machines with more than two pole-pieces.

$r$, outside radius of armature.

$\mathrm{L}$, length of armature parallel to the axis.

$k$, a constant such that $k r \mathrm{~L}=a_{1} ; k$ is as much as 1.25 in drum or Hefner-Alteneck armatures, and as little as 0.5 in short cylindric or other Gramme armatures.

$t$, the thickness of the winding of the armature.

$d$, the clearance between wire and pole-piece.

$\delta=d+t$, the total distance from iron of armature to polepiece.

$n$, the revolutions per second of the armature.

$v$, the circumferential velocity of the armature in centimetres per second $v=2 \pi r n$.

$\mathrm{S}$, wires counting all round the outside of the armature.

$A$, amperes in each wire.

$\mathrm{SA}$ will be called the "ampere-wires" on the armature, being the ampere-turns if it is a Gramme, and being twice the ampere-turns if it is a Hefner-Alteneck.

$a$, amperes per sq. centim. flowing in the section of the armature-winding made by a plane at right angles to the axis.

$\alpha_{1}$, the highest permanent value of $\alpha$ allowable.

$\rho$, the electric resistance of the armature-winding per cubic centimetre. That is, per centimetre of its length per sq. cm. of cross section. Of course $\rho$ is greater than the specific resistance of copper, as the space is partly occupied by insulating material, $\rho$ is greater with finer wire; but for some practical purposes it is advisable to assume $\rho$ constant for all kinds of winding.

$\rho \alpha^{2}$, watts developed as heat per cubic centimetre of the winding, so that total rate of development of heat $=2 \pi r t \mathrm{~L} \rho \alpha^{2}$.

$a_{1}$, cross section of iron of armature by a diametral plane.

$a_{2}$, area of pole-piece exposed to the armature, increased by a fringe of $0.8 \delta$ in breadth all round.

$a$, the cross section of any other portion of the magnetic circuit which may be considered.

$\beta$, the induction in C.G.S. units, given in lines per square centimetre anywhere in the magnetic circuit. 
$\beta_{1}$, the value of $\beta$ in the iron of the armature.

$\beta_{1}{ }^{\prime}$, the greatest value of $\beta$ which it is convenient to employ in the armature when the machine is giving its greatest permanent output.

$\mathrm{N}$, the total induction in the iron of the armature $\mathrm{N}=\beta_{1} a_{1}$ or $\beta_{1} k r \mathrm{~L}$.

$\frac{2 \delta}{a_{2}}$ or $\frac{2(t+d)}{a_{2}}$ will be called the magnetic air-resistance, a not very incorrect or misleading expression.

$\frac{l \nu}{a \mu}$ will be called the magnetic resistance of a portion of the iron part of the magnetic circuit whose length is $l$, whose cross section is $a$, the permeability being $\mu$, $\mathrm{N} \nu$ being the total induction there.

The whole iron magnetic resistance may be written $\Sigma \frac{l \nu}{a \mu}$.

This is for only one of the magnetic circuits if the machine has more than one.

In a well-designed modern machine there is no throttling of the induction anywhere, and $\frac{l}{a_{1} \mu}$ may be taken as the whole magnetic iron resistance, $\mu$-being the permeability of the iron of the armature, $l$ the average total length of a line of induction in the iron of the whole machine.

W watts, the power developed by the rotation of the armature.

$\mathrm{W}^{\prime}$, the permanent highest power developed.

$\mathrm{E} \theta$, the rate of loss of heat in watts per square centim. of the cylindric outer surface of the armature. This is usually taken to be 0.2 in modern machines furnished with some ventilating arrangement. The value of $\sqrt{\frac{\mathrm{E} \theta}{\rho}}$ may, we find, be practically taken as 288 in such machines; we shall call this constant $q$, as machines made by different makers differ greatly in its value, even where there are the same methods of ventilating and the same sizes of wires. With finer wires $\rho$ is greater, as the space is less occupied by copper, so that $q$ is less. If two thirds of the space be taken as occupied by copper, $q=288$ if $\mathrm{E} \theta=0 \cdot 2$. In some armatures, which are always perhaps too safe from heating, we have found $q$ to be as little as 150 . In some cases it is considerably more than 300 . 
$\mathbf{S}^{\prime}$, spires on the coils of the field-magnet part of one magnetic circuit. In the Edison-Hopkinson and Kapp machines there is only one magnetic circuit, and $\mathrm{S}^{\prime}$ is here the total number of spires on the field-magnets; but in the Manchester, Crompton, A-Gramme, and other forms there are two magnetic circuits, and $\mathbf{S}^{\prime}$ is here the number of spires on only one of the magnetic circuits.

$\mathbf{A}^{\prime}$, amperes in each spire of the field magnet-coils ; or

$\alpha^{\prime}$, amperes per square centimetre of cross section of the winding.

Any person who has engaged in making measuring-instrunents or dynamo machines is aware that for a given volume of winding, whether the wires are small or large, for the same distribution of temperature there will be the same number of ampere-turns and there will be the same rate of loss of energy by heating, if the volume of insulating material is always in the same ratio to the volume of the copper. With fine wire the volume of insulating material becomes greater, but not to such an extent as to make useless this very important roughly correct practical rule for the makers of instruments and dynamo machines. This rule we have regularly used since 1881 in our measuring-instruments.

This has led us to speak of $\alpha$ the current in amperes per square centimetre of cross section of a coil, rather than the density of current in the copper alone, and we have been led to some general rules of considerable practical interest in consequence.

To make our rules more complete, we begin with one which is well known. It will be observed that we use Mr. Kapp's method of counting wires on the armature, so as to make the rule suitable both for the Hefner-Alteneck and the Gramme armature.

$$
\begin{aligned}
& \text { Total E.M.F. of armature }=\frac{n S N}{10^{8}} \ldots . . . \\
& \left.\begin{array}{l}
\text { E.M.F. developed in unit length } \\
\text { of wire passing through the field }
\end{array}\right\}=\frac{v k \beta_{1}}{\pi 10^{8}} \quad . .
\end{aligned}
$$

Observe that it is only the wire on the convex outer surface of the armature which is here considered.

$$
\begin{aligned}
& \mathrm{W}=\frac{2 n \mathrm{~N}}{10^{8}} \mathrm{SA} . \quad . \quad . \quad . \quad . \\
& \mathrm{W}=\frac{2 v \mathrm{~N} t \alpha}{.10^{8}} . \quad . \quad . \quad . \quad . \quad .
\end{aligned}
$$


The heat generated in the armature-winding per cubic centim. $=\rho \alpha^{2}$. The possible ventilation arrangements are better in Gramme than in Hefner-Alteneck armatures, and it is usual, therefore, to consider only the heat generated in the wires on the outside convex surface of the iron of either. This is $2 \pi r t \mathrm{~L} \alpha_{1}^{2} \rho$; and as the heat emitted is found, for the highest temperature $\theta$ at which it is considered safe permanently to work the machine, to be proportional to the convex surface $2 \pi r \mathrm{~L}$, if $\mathrm{E}$ is the rate of loss of heat in watts per square centimetre of this surface,

$$
2 \pi r t \mathrm{~L} \rho \alpha_{1}^{2}=\mathrm{E} \theta 2 \pi r \mathrm{~L}
$$

or

$$
t \rho \alpha_{1}^{2}=\mathrm{E} \theta \text {. }
$$

We find that $\frac{\mathrm{E} \theta}{\rho}$ may be taken as 83000 in the best modern machines; we shall call this $q^{2}$, so that

$$
t \alpha_{1}^{2}=q^{2} \text {. . . . . . . . }
$$

We can, therefore, from (4), express the greatest permanent output of a machine $W^{\prime}$, either in terms of $\alpha_{1}$ or of $t$ :

$$
\begin{aligned}
& \mathrm{W}^{\prime}=0.00166 \frac{v \mathrm{~N}^{\prime}}{\alpha_{1}}, \text {. . . . . . . } \\
& \mathrm{W}^{\prime}=\frac{2 q}{10^{8}} v \mathrm{~N}^{\prime} \sqrt{t}, \ldots . \quad . \quad . \quad . \quad . . \\
& \mathrm{W}^{\prime}=\frac{2 g}{10^{8}} v k r \mathrm{~L} \beta_{1}{ }^{\prime} \sqrt{t}, \ldots . . . .
\end{aligned}
$$

$\mathrm{N}^{\prime}$ and $\beta_{1}^{\prime}$ being the highest allowable values of $\mathrm{N}$ and $\beta_{1}$. It is on the combination of (5) and (4) that we have based an important generalization regarding the magnetic circuit of the dynamo, which gives the title to our paper. Members of the Society are aware of the methods adopted by Mr. Bosanquet, Dr. Hopkinson, and Mr. Kapp in dealing with a magnetic circuit. Consider a closed tube of small cross section everywhere, passing through the iron of the armature, the air-spaces, the limbs of the field-magnet, and the yoke. Let the section vary so that the total induction is everywhere the same. Then, in a short length of the tube $l$, the lineintegral of the magnetic force required to produce this induction is

$$
\frac{l}{\mu} \beta
$$

where $\mu$, the magnetic permeability of the material at any place, is 1 for air, and where. its value for iron is given in 
the accompanying figure for various values of $\beta$. The curve $\mathrm{B}$, fig. 1, is that given by Mr. Bidwell in his paper (Proc. Royal Society, No. 245, 1886). The curve $\mathrm{H}_{1}$ has been obtained from Dr. Hopkinson's experiments on wrought iron in the Philosophical Transactions, 1885, plate 47. We wish that Dr. Hopkinson had in this paper given his experimental numbers, instead of plotting them in curves to such scales that one feels very great uneasiness in making measurements. We have determined the curve $\mathrm{H}_{1}$ by taking a medium curve between Dr. Hopkinson's curves for rising and falling magnetizations. It is quite probable that, in view of the great differences in the behaviour of the same and different kinds of iron, under different circumstances, Dr. Hopkinson did not feel that he was justified in drawing his curves on paper too finely divided. It will be observed that from $\beta=10000$ to $\beta=16300$ we may take $\mu=5221-0.3071 \beta$. Unfortunately for easy methods of calculation in many machines much larger values of $\beta$ than 16300 are often used.

Curve $\mathrm{H}_{2}$ is computed from Dr. Hopkinson's results for cast iron, and it shows how very important it is, when cast iron is used in the magnetic circuit of a machine, to have its section much greater than that of the wrought iron portions of the circuit, if throttling the induction is to be avoided.

Curves $K_{1}, K_{2}$, and $K_{3}$ are computed from the formulæ given in Mr. Kapp's paper for the wrought iron used in the armature, field-magnets, and yoke respectively of a dynamo machine.

The differences among these curves throw great light upon the fact that it is really impossible to predetermine the "characteristic" of a dynamo machine. Mr. Kapp gets over discrepances by calculating a value of his leakage-resistance which suits the actual observations made on an already constructed dynamo machine; and Dr. Hopkinson's curves, although calculated on the basis of actual measurements of the leakage made on an already constructed dynamo, and although only computed for a very small portion of that part of the characteristic where the iron magnetic resistance is important (the only portion about which there is any difficulty), represent this small portion very indifferently indeed. Methods of calculating the leakage which have been put forward we can in no way believe in, for reasons given by one of us in the discussion on Mr. Kapp's paper at the Society of Telegraph Engineers. We are not in any way detracting from the merits of Dr. Hopkinson's beautiful theory. We are too well aware of the great services he has done us and of the

Phil. Mag. S. 5. Vol. 25. No. 157. June 1888. $2 \mathrm{~L}$ 
enormous change which he has produced in the construction of dynamos.

Now the line-integral of the magnetic force round a closed magnetic circuit-called by Mr. Bosanquet the Magnetomotive Force-is the number of ampere-turns on the coils through which it threads it way $\times \frac{4 \pi}{10}$. Hence if $\mathrm{S}_{2} \mathrm{~A}_{2}$ be the ampere-turns on the field magnet,

$$
\frac{4 \pi}{10} \mathrm{~S}_{2} \mathrm{~A}_{2}=2 \delta \beta+\Sigma \frac{l}{\mu} \beta, . . \quad . \quad .
$$

$\delta$ being $d+t$ the distance from iron of armature to iron of field-magnet, and of course the length of the tube in air is twice this distance as it goes into and out of the armature.

If $\mathrm{N}$ is the total induction through the iron of the armature, $\frac{N}{a_{1}}=\beta$ there and $\frac{N}{a_{2}}=\beta$ in the air-space.

If $v \mathrm{~N}$ is the total induction through the iron of the fieldmagnet anywhere, then $\frac{\nu \mathrm{N}}{a}=\beta$ there, so that (9) may be written

or

$$
\begin{aligned}
& \quad \frac{4 \pi}{10} \mathrm{~S}_{2} \mathrm{~A}_{2}=2(d+t) \frac{\mathrm{N}}{a_{2}}+\Sigma \frac{l \nu \mathrm{N}}{a \mu}, \\
& \mathrm{N}=\frac{\frac{4 \pi}{10} \mathrm{~S}_{2} \mathrm{~A}_{2}}{\frac{2(d+t)}{a_{2}}+\Sigma \frac{l \nu}{a \mu}} ; . . . . . . .
\end{aligned}
$$

and as $\mathrm{N}$ is the induction through the armature produced by a magneto-motive force $\frac{4 \pi}{10} \quad \mathrm{~S}_{2} \mathrm{~A}_{2}$, equation 10 leads to $\frac{2(d+t)}{a^{2}}$ being called, by analogy with other physical resistances, the air magnetic resistance, and $\Sigma \frac{l_{\nu}}{a \mu}$ the iron magnetic resistance of the circuit. The members of the Society will perhaps allow us to put in this way Dr. Hopkinson's theory, although he himself may object to some of the terms we use.

Now let it be assumed that there is a value of $\beta$ for the armature iron which it is best to use in all machines when giving their permanent output. $\nu$ may also be taken as practically constant. We are aware that to both these assumptions exception may be taken, but this will not be found to affect the practical general result which we arrive at. 
Then $\mu$ is known, so that if the lengths and cross sections of the iron everywhere are known, the iron magnetic resistance $\Sigma \frac{l v}{a \mu}$ is known. Inserting this value of $\mathrm{N}^{\text {as }} \mathrm{N}^{\prime}$ in (7) we find

$$
\mathrm{W}^{\prime}=\frac{2 q}{10^{8}} v \sqrt{t} \frac{\frac{4 \pi}{10} \mathrm{~S}_{2} \mathrm{~A}_{2}}{\frac{2(d+t)}{a_{2}}+\Sigma \frac{l \nu}{a \mu}} ; .
$$

and it will be found that this is a maximum for different values of $t$ when

$$
\frac{2 t}{a_{2}}=\frac{2 d}{a_{2}}+\Sigma \frac{l \nu}{a \mu}, \ldots . . .
$$

That is, when the magnetic resistance of the space occupied by the winding of the armature is equal to the resistance of the rest of the magnetic circuit.

When this is the case (11) reduces to

$$
\mathrm{W}^{\prime}=\frac{2 \pi q}{10^{8}} \frac{v a_{2}}{\sqrt{t}} \mathrm{~S}_{2} \mathrm{~A}_{2} . \ldots .
$$

That the ampere-turns on the field-magnet $S_{2} A_{2}$ may really produce the given induction $\beta_{1}$ in the armature, it is necessary that

$$
\mathrm{S}_{2} \mathrm{~A}_{2}=\frac{10}{\pi} \frac{a_{1} t}{a_{2}} \beta_{1} ;
$$

and if we insert this value in (13) we have of course (7) again.

It is now the custom in making dynamos to let $\beta$ be nearly constant in all parts of the circuit where the iron is the same, and to have $\frac{\beta}{\mu}$ as nearly constantas possible, if different kinds of iron are employed. If $\frac{\beta}{\mu}$ is larger anywhere than its average value for the rest of the circuit, we say that the induction is there "throttled," and throttling is only allowable in the armature, if it is allowable anywhere. If $\frac{\beta}{\mu}$ is nearly constant everywhere in the iron, we may take $\frac{l}{a_{1} \mu}$ as the total iron resistance of the circuit, $l$ being the average length of the lines of magnetic induction in the iron of the whole circuit, and then we may use

$$
\frac{l}{a_{1} \mu} \text { instead of } \Sigma \frac{l \nu}{a \mu}
$$

$2 \mathrm{~L} 2$ 
in $(10),(11)$, and (12); $\mu$ being the permeability of the armature iron when the induction is $\beta_{1}{ }^{\prime}$.

In the above investigation, the iron resistance is supposed to be given, and also the exciting power in ampere-turns. At present this seems a sufficiently practical basis for the calculation, as we usually fix first the size of armature, then arrange as short a magnetic circuit as possible, which must leave sufficient room for the exciting coils. We see, however, that a larger generalization is possible when we know with certainty what is the limiting thickness of winding on the field-magnets. We were allowed to assume in the armature winding that $t \alpha_{1}{ }^{2}$ is constant, $\alpha_{1}$ being the greatest permanent current per square centimetre allowable. When the winding is thin, so that the temperature is nearly uniform in the winding, and only when this is the case, is such a rule allowable, and it is not allowable in cases where the winding is of the thickness usual in field-magnet coils. We are at present experimenting on this subject, but there are considerable difficulties in the way of obtaining practical rules. We have no doubt, however, that there is such a rule as

$$
\mathrm{S}_{2}{ }^{\prime} \mathrm{A}_{2}^{\prime}=p \lambda \text {. }
$$

That is, the greatest number of ampere-turns which can usefully and permanently be applied on a field-magnet coil of length $\lambda$ is proportional to $\lambda$. If $l$ is given, the configuration of the machine enables $\lambda$ and therefore $S_{2}^{\prime} A_{2}^{\prime}$ to be fixed. Then $\mathrm{S}_{2}{ }^{\prime} \mathrm{A}_{2}{ }^{\prime} \div \frac{2 l}{\mu_{1}}=\beta_{1}$ enables $\beta_{1}$ and $\mu_{1}$ to be calculated by trial if there is sufficient information about the character of the iron. $\frac{2 t}{a_{2}}$ is now made equal to $\frac{2 d}{a_{2}}+\frac{l}{a_{1} \mu_{1}}$, so that the important dimensions are fixed. Should $\beta_{1}$ be great, considerations of cost of the field-magnet winding may come in, to alter completely the design of the machine, but for a given configuration of machine this is the practical method of working.

From experiments, the results of which were published before this Society on March 12th, 1887, we came to the conclusion that there is a definite resistance at a joint in a magnetic circuit ; and Professor Ewing found that by cutting a bar into two, four, and eight pieces, the magnetic permeability seemed to alter from 1220 to 980,640 , and 480 respectively, the joints being tooled up in the usual way. When the joints are carefully scraped he also found that they materially increased the magnetic resistance unless considerable pressure was applied. Now as a stress of less than one quarter of the ordinary stresses to which wrought iron is 
subjected in machinery materially diminishes the permeability of iron, it is obvious that all joints in the magnetic circuit ought, as much as possible, to be got rid of, consistently with easy manufacture; or, when they exist, they ought to be made of as large area as possible, by letting one piece into another to a considerable depth.

In the design of a machine, the question of possible leakage is of considerable importance. It will often be found that in the effort to make $l$ short and $a_{2}$ large, machines are designed with enormous amounts of leakage not only from one pole to the other, but from either pole to the middle of a neighbouring field-magnet coil.

One of the best ways of finding the nature of the probable magnetic leakage in a dynamo, before it is constructed, is to construct a small model of the same kind of iron, exciting the field-magnets, and exploring by means of a ballistic galvanometer. Another simpler way, which gives a considerable amount of information, and which we have employed, is to make a model of wood, covering certain judiciously selected parts, such as the poles and armature, and half the fieldmagnets with metal, immerse the model in a barrel of rainwater, and find the electric resistance between one part and another when electric potential-differences are established between them. On account of the ease with which the model may be rearranged in configuration, this method of working gives interesting results ; but these results, when applied in the magnetic case, must be used judiciously and with the knowledge that the permeability of iron is not a fixed quantity.

It is obvious that the best section of a field-magnet limb is the circular, but considerations of possible leakage to the middle of the limb from the armature or a pole-piece, and other considerations relating to the configuration of the machine, often cause us to give to the section a rectangular or oval section.

\section{The Characteristic of a Dynamo.}

In a letter written by one of us from Japan, in January $1879^{*}$, before he had seen a dynamo, the necessity was shown for establishing an algebraic relation between the E.M.F. developed in the armature and the current exciting the fieldmagnets. It was pointed out that $E$ the E.M.F. in the armature was proportional to the field and speed, and that this led to

$$
\mathrm{E}=p+q c+\Sigma \frac{a c}{b+c} \cdot \text {. . . . }
$$

* Not published till 1885, and then in a somewhat mutilated form in the 'Electrician' of November 20. 
for any given speed. It was also pointed out that a tangent function might also be employed, but that it would not lend itself so readily to calculation. We had, both, in 1878 used a curve to express this relationship, but it was not until 1881, when we met M. Deprez, and learnt of his work, that we had any conception of the many calculations which might be made by graphical methodz, using the curve as a fundamental relation. Herr Fröhlich uses one term of the above expression:-

$$
\mathbf{E}=\frac{a c}{b+c} \cdot \cdot \cdot \cdot \cdot \cdot \cdot(15)
$$

He regards $p$ the permanent field as 0 , and he has no term which remains proportional to the current. We shall speak of (15) as the Fröhlich formula, because it usually goes under that name. It is, of course, an empirical formula, like (14).

The reasoning which led us to regard (14) as a rational formula, was based on a magnetic theory which need not here be expounded.

A very great necessity exists for having some such empirical formula as (15), but, as is well known, it is quite incorrect when $c$ is small. It is not nearly so incorrect as we might imagine it to be from the statements made by Mr. Kapp (p. 529, Journ. Soc. Tel. Eng. and Elect. vol. xv. 1887), who has given in his fig. 3 a most absurdly unsuitable Fröhlich curve, or by Dr. Hopkinson (plate xvi. Phil. Trans. 1886), whose Fröhlich is also not the most suitable. It is obvious that the Fröhlich ought not to be expected to agree with the real characteristic near the origin. It ought to be made to agree most perfectly with the working part of the characteristic. To effect this purpose let the observed values of $\frac{\mathrm{E}}{\mathrm{U}}$ and $\mathrm{E}$ be plotted as the coordinates of points on squared paper. The straight line which lies most evenly among the points for the working values of $\mathrm{E}$ satisfies the equation

$$
\frac{\mathrm{E}}{\mathrm{C}} b+\mathrm{E}=a \text {. }
$$

The measured coordinates of two points on this straight line enable $b$ and $a$ to be calculated.

Thus, for example,

$$
\mathrm{N}=\frac{1364 \mathrm{~F}}{1+2 \cdot 7 \times 10^{-4} \mathrm{~F}}
$$

will be found to satisfy the observations published by the Drs. Hopkinson for the Manchester dynamo from $\mathrm{F}=6000$ to $\mathrm{F}=30,000$ with a wonderful amount of accuracy. But for 
the early part of the curve it is quite unsuitable. $F$ is $\frac{4 \pi}{10} \times$ ampere-turns in one coil of the machine, or the magnetomotive force.

The uses made by Herr Fröhlich and Prof. Rücker of (15) in a theory of compounding show how important it is to have a simple empirical formula. Indeed in our patent specification of 1882 we base the theory of compounding on the simplest of all empirical formulæ,

$$
\mathrm{E}=a \mathrm{C} . \text {. . . . . . . }
$$

And it is by means of this formula that the theory can be best put before students ; if our easy reasoning is properly grasped, the practical electrical engineer will be able to use the results graphically with actual characteristics in the manner employed by the most experienced men at the present day.

Instead of speaking of $\mathrm{E}$ the E.M.F. produced in the armature, which is proportional to $\mathrm{N}$ the magnetic field and $n$ the speed, as shown in (1) it is preferable to speak of $\mathrm{N}$ the field itself. And instead of referring merely to the current exciting the field-mignets, it is preferable to speak of the ampereturns $\mathrm{S}_{2} \mathrm{~A}_{2}$, or, better, of the magneto-motive force in C.G.S. units, $\frac{4 \pi}{10} \mathrm{~S}_{2} \mathrm{~A}_{2}$.

We have tried in vain, during perhaps two months of very hard work, to express the two constants $a$ and $b$ of (15) in terms of the dimensions of a dynamo machine. We now believe that this cannot be effected. We have already given the reason why the linear law connecting the $\beta$ and $\mu$ cannot be used in obtaining a formula. In any case such a formula would be very different from that of Fröhlich. But in view of the important relation (12) which we have established, that when the machine is giving its best permanent output,-

The air magnetic resistance of the space occupied by winding on the armature = all other magnetic resistance,

it is not difficult to arrive at practical rules of working. It will be noticed that in existing machines we may, in general calculations, neglect the resistance of the clearance-space $d$.

The most important fact to be kept in mind by constructors of dynamos is, that the magnetic air-resistance is the governing factor. Until half the highest induction is reached we may neglect altogether the resistance of the iron; and indeed for many calculations it is sufficient to take

$$
\mathrm{N}=\frac{4 \pi}{10} \frac{a_{2}}{2 \delta} \mathrm{S}_{2} \mathrm{~A}_{2} ; . . . . . .
$$


where $a_{2}$ is the area of the pole-piece exposed to the armature (increased by the fringe-area, which Dr. Hopkinson estimates to be of a breadth $0 \cdot 8 \delta$ ), and $\delta$ is the distance from iron of armature to iron of pole-piece.

(17) gives, in fact, the straight part of the characteristic. In well-constructed dynamos the magnetic iron resistance is equal to the air resistance $\frac{2 \delta}{a_{2}}$ when the machine is working at its most permanent output ; and it is only necessary to know $\beta^{\prime}$, the induction convenient to use when this is the case, to be able to calculate $\mathrm{N}$ and $\mathrm{S}_{2} \mathrm{~A}_{2}$ for a point on the curved part of the characteristic. Thus

$$
a_{1} \beta_{1}=\mathrm{N}_{1}=\frac{4 \pi}{10} \frac{a_{2}}{4 \delta} \mathrm{S}_{2} \mathrm{~A}_{2}
$$

so that if $\alpha_{1}$ and $\beta_{1}$ are known, $\mathrm{S}_{2} \mathrm{~A}_{2}$ can be found. Thus in the Manchester dynamo described by Dr. Hopkinson (Trans. Roy. Soc. 1886),

$$
\begin{aligned}
& a_{1}=220.5 \text { square centim., } \\
& a_{2}=839.5 \quad, \quad, \\
& \delta=0.8 \text { centim. }
\end{aligned}
$$

Taking $\beta_{1}=18,460$ lines per square centim., we find

Now

$$
a_{1} \beta_{1}=4.07 \times 10^{6} \text {. }
$$

$$
\frac{4 \pi}{10} \frac{a_{2}}{2 \delta}=660 \text {. }
$$

So that $\mathrm{N}=660 \quad \mathrm{~S}_{2} \mathrm{~A}_{2}$ represents the straight part of the characteristic.

$$
\begin{array}{ll}
\text { Again, } & \mathrm{N}_{1}=a_{1} \beta_{1}=4.07 \times 10^{6}, \\
\text { and } & a_{1} \beta_{1}=330 \mathrm{~S}_{2} \mathrm{~A}_{2}, \\
\text { or } & \mathrm{S}_{2} \mathrm{~A}_{2}=\frac{4.07 \times 10^{6}}{330}=12330 .
\end{array}
$$

Plotting $\mathrm{N}=4.07 \times 10^{6}$ and $\mathrm{S}_{2} \mathrm{~A}_{2}=12330$ as the coordinates of a point on squared paper, and plotting also the straight line from the origin $\mathrm{N}=660 \mathrm{~S}_{2} \mathrm{~A}_{2}$, a man who has seen characteristics before, will be able to draw the characteristic of this machine with a fair amount of accuracy, especially if he recollects that the iron is near saturation. Those, however, who have less experience may find the Fröhlich, which for any dynamo passes through the point on the straight line which represents. $N=10,000 a_{1}$, and the second point just found, 
rounding off the straight line into the Fröhlich by hand. In applying our rule to the Manchester dynamo we employed neither of these methods, as one's judgment is vitiated when one has seen the actual observations; and we have assumed that the very roughly correct rule,

$$
\mathrm{N}=p \tan ^{-1}\left(h \cdot \mathrm{S}_{2} \mathrm{~A}_{2}\right),
$$

may be applied, making its slope the same as that of the straight line at the origin, and making it pass through the point whose position we have calculated. In this case

$$
p h=660 ;
$$

and it will be found that

and

$$
p=34 \cdot 65 \times 10^{5},
$$

for this case.

$$
h=19 \cdot 03 \times 10^{-5}
$$

As Dr. Hopkinson has given his characteristics, not in terms of ampere-turns $\mathrm{S}_{2} A_{2}$, but in absolute units, calling $\frac{4 \pi}{10} \mathrm{~S}_{2} \mathrm{~A}_{2}$ by the letter $\mathrm{F}$ to denote the magneto-motive force in absolute units, the above formula becomes for this comparison,

$$
\mathrm{N}=34 \cdot 65 \times 10^{5} \tan ^{-1}\left(15 \cdot 15 \times 10^{-5} \mathrm{~F}\right) \text {. }
$$

In the figure we have plotted the curve obtained by calculation as A; B shows Dr. Hopkinson's calculated curves; the dotted line passes as nearly as possible through the points determined by experiment. It will be observed that the Drs. Hopkinson have only ventured to calculate the characteristic up to $\mathbf{F}=12,000$ on the ascending, and 9500 on the descending part; whereas the observations extend to $\mathrm{F}=29,500$. As the very straight part near the origin presents no difficulty, it may be said that all their elaborate calculation represents only the characteristic from about $\mathrm{F}=6000$ to $\mathrm{F}=12,000$, and that part very indifferently. It is, besides, to be observed that in an actual dynamo there are no such differences between observations made with steadily increasing and with steadily diminishing magneto-motive forces, as the theory requires.

In the above calculation we have neglected the clearance $d$, as we did not know it. It would have been easy to calculate a value of $d$, possibly not very different from the real value, to make our curve agree more closely with the observations. In conclusion we would say that, in our opinion, it is impossible, until a machine is constructed, to compute its characteristic 
with sufficient accuracy for such purposes as the determination of coils in compound winding. But the probable characteristic may be determined in the way here described, with sufficient accuracy for a number of useful purposes. It is based on the facts:-(1) When a machine is working at its best permanent output, its iron magnetic resistance plus the air magnetic resistance of the clearance is equal to the air magnetic resistance of the space on the outside of the armature occupied by winding. (2) At the beginning the airresistance is alone of any importance.

LXII. On the Recalescence of Steel. By H. F. Newald, M.A., Demonstrator in Experimental Pliysics, Cambridge University*.

TN a Note published in the Philosophical Magazine for 1 November 1887, I stated the results of a number of experiments made with a view to investigate the recalescence of steel. I propose in the present note to give an account of the experiments on which I base those results.

I. Reglow, or recalescence, is not due to chemical action at the surface of the steel, nor is it due to occlusion of gases.

A steel wire with copper terminals was put in a glass tube, the terminals passing through indiarubber stoppers, which closed the tube tightly at its two ends. The tube was exhausted by means of a mercury-pump till the air-pressure was a very small fraction of a millimetre. The steel wire was heated again and again by the passage of an electric current from storage-cells. Pressure rose slightly after the first three or four heatings (probably because of the setting free at high temperatures of the air-layers condensed on wire and tube surfaces), and was reduced by further pumping. Both darkening and reglow were observed with undiminished distinctness after fourteen heatings, and the process was not repeated. Nitrogen was admitted to the tube and produced no effect that could be detected on the brilliancy of reglow.

II. Reglow is not due to difference in conductivity at different temperatures, as suggested by Forbes.

A wire ( .5 millim. diameter) was hammered flat and still showed reglow. A thin steel plate, hammered till its thickness was less than 1 millim., as measured by a screw-gauge, still showed reglow. Either we must believe that there can

* Abstracted from a paper read before the Cambridge Philosophical Society, January 29, 1888. Communicated by the Author. 


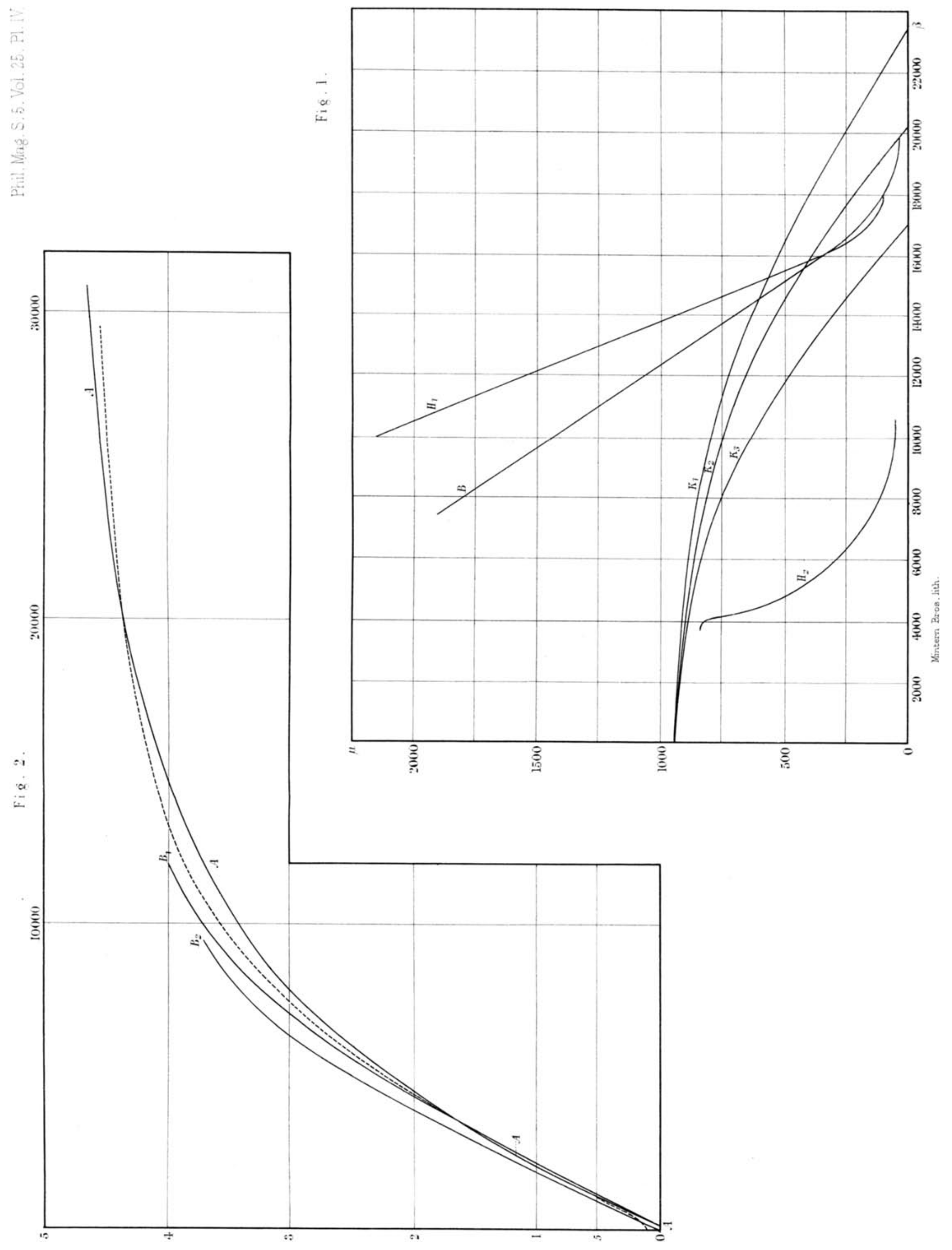

